lockdown (67\%). Singles who continued to have sex more often reported casual regular partner during the first (58\%) and second lockdown (60\%), than pre lockdown (28\%) and between lockdowns (33\%). STI testing uptake decreased from $9 \%$ pre-lockdown to $1 \%$ during the first and $3 \%$ during the second lockdown. Self-tests were used almost twice as often during the pandemic (13\%) as before (7\%). Among the reasons for not testing, corona-related reasons (e.g., STI clinics are closed, or health care providers are too busy) decreased between the first (47\%) and second lockdown (27\%). Furthermore, youths mental health has weakened since the first lockdown up until the end of 2020 .

Conclusion COVID-19 pandemic significantly impacts the sexual health of Dutch youth, especially during lockdown periods, but also in periods of relaxation of pandemic control measures. Therefore, it is extremely important for healthcare providers to make their facilities accessible and find for alternatives for face-to-face testing.

\section{P345 TEST AND TREAT, OR TEST AND WAIT?: MANAGING SEXUAL PARTNERS AT RISK FOR STI}

${ }^{1} \mathrm{~F}$ van den Heuvel ${ }^{*},{ }^{1} \mathrm{E}$ Tahar-Kooijman, ${ }^{1,2} \mathrm{H}$ de Vries, ${ }^{1} \mathrm{~T}$ Heijman. ${ }^{1}$ Public Health Service of Amsterdam, Department of Infectious Diseases, Amsterdam, The Netherlands; ${ }^{2}$ Department of Dermatology, Amsterdam Institute for Infection and Immunity (AlandII), Amsterdam University Medical Centers, location Academic Medical Centre, University of Amsterdam, Amsterdam, The Netherlands

\subsection{6/sextrans-2021-sti.393}

Background Treatment of sexual partners notified for Chlamydia trachomatis(CT) or Neisseria gonorrhoeae (NG) is needed to prevent ongoing transmission. To prevent the emergence of Anti-Microbial resistance, antibiotics should be used prudently. Therefore, in 2020, the STI clinic of the Public Health service of Amsterdam (STI clinic) went from treating all confirmed notified sexual partners (before test results) to a selection of the notified partners i.e. only those who received confirmed notification from a steady sex partner, with whom they had sex in the last 2 months. We evaluated STI outcomes before and after implementation of this policy.

Methods Routine data from confirmed notified partners attending the clinic for CT/NG from 01-09-2019 to 01-032020 were compared with similar data from 01-03-2020 to 01-10-2020.

Results CT: of the 132 partners notified before policy change, $64 \%(84 / 132)$ had a negative test result, all received treatment before results were available. After policy change 71\% (163/ 230) had a negative result, $17 \%(39 / 230)$ of them received treatment before results were available and 14\% (32/230) positive for CT had to return to the clinic to receive treatment.

NG: of the 131 partners notified before policy change $72 \%(94 / 131)$ had a negative test result, all received treatment before results were available. After policy change 71\% (215/ $304)$ had a negative test result, 27\% (59/215) of them received treatment before results were available and 57\% (51/ 89 ) of the patients positive for NG had to return to the clinic for treatment.

Discussion Unnecessary treatment with antibiotics decreased substantially when introducing the new policy. A limited amount of partners had a delay in treatment and had to return to the clinic. The cost of a second visit and the delay to treatment will be assessed in order to improve services and decrease the risk of distribution of CT/NG by untreated partners.
P346 REACHING SEXUAL PARTNERS AT RISK FOR STI THROUGH SOCIAL MEDIA

${ }^{1}$ E Tahar-Kooijman*, 'F van den Heuvel, ${ }^{1,2} \mathrm{H}$ de Vries, ${ }^{1} \mathrm{~T}$ Heijman. ${ }^{1}$ Public Health Service of Amsterdam, Department of Infectious Diseases, Amsterdam, The Netherlands; ${ }^{2}$ Department of Dermatology, Amsterdam Institute for Infection and Immunity (AlandII), Amsterdam University Medical Centers, location Academic Medical Centre, University of Amsterdam, Amsterdam, The Netherlands

\subsection{6/sextrans-2021-sti.394}

Background At the STI clinic of the Public Health service of Amsterdam (STI clinic) partner notification (PN) seems successful in the early diagnosis and treatment of STI's in sexual partners. Together with the healthcare staff, and upon consent of the index patient, sexual partners at risk are identified and the PN methods discussed: patient or provider referral, by phone, mail or an online PN service. However, in a substantial number of cases, contact details are missing because of the anonymity of the partner, e.g in case of social media (SM) use. We therefor explored the willingness and opinion of patients to use SM for online PN.

Methods From December 172020 to February 12021 we invited all patients visiting the STI clinic for treatment to fill in an anonymous online questionnaire on the use of SM for online PN: 1) using SM for meeting and notifying sexual partners, and 2) to be approached themselves as a notified partner. Lastly, they were asked to comment on a series of potential texts messages to be used for online PN.

Results 180 patients participated in the study: 68\% males, $32 \%$ females and $0,6 \%$ transgender persons. $80 \%$ indicated they met a sexual partner via SM and $57 \%$ of them only had SM contact details. 59\% would like to use a message via SM for PN purposes, and $73 \%$ would want to receive a PN via SM. $40 \%$ would want to be informed when involving HIV. The majority would choose a format containing the logo of the public health service and the online PN service because this appeared more trustworthy.

Discussion Preliminary analyses show the potential of online PN through SM. However safety and trustworthyness are concerns when developing the intervention and effectiveness should be analysed.

\section{P347 DEVELOPMENT AND EXTERNAL VALIDATION OF A PROGNOSTIC MODEL FOR SURVIVAL OF PEOPLE LIVING WITH HIVIAIDS INITIATING ANTIRETROVIRAL THERAPY}

1,2J Wang, ${ }^{3} \mathrm{~T}$ Yuan*, ${ }^{4} \mathrm{H}$ Ding, ${ }^{4} \mathrm{~J} \mathrm{Xu},{ }^{1} \mathrm{~W}$ Keusters, ${ }^{5} \mathrm{X}$ Ling, ${ }^{3} \mathrm{~L} F u,{ }^{3} \mathrm{Q} Z \mathrm{Zhu},{ }^{5} \mathrm{Q} \mathrm{Li},{ }^{5} \mathrm{X}$ Tang, ${ }^{5} \mathrm{~W}$ Cai, $6,7,8,9 \mathrm{H}$ Hong, ${ }^{5} \mathrm{~L} \mathrm{Li}, 3,10 \mathrm{H}$ Zou. ${ }^{1} J u l i u s$ Center for Health Sciences and Primary Care, University Medical Center Utrecht, Utrecht, The Netherlands; ${ }^{2}$ Division of Pharmacoepidemiology and Clinical Pharmacology, Utrecht Institute for Pharmaceutical Sciences, Utrecht University, Utrecht, The Netherlands; ${ }^{3}$ School of Public Health (Shenzhen), Sun Yat-sen University, Shenzhen, China; ${ }^{4}$ NHC Key Laboratory of AIDS Immunology, Department of Laboratory Medicine, First Affiliated Hospital of China Medical University, Shenyang, China; ${ }^{5}$ Eighth People's Hospital, Guangzhou Medical University, Guangzhou, China; ${ }^{6}$ Key Laboratory of AIDS Immunology of National Health Commission, Department of Laboratory Medicine, the First Affiliated Hospital of China Medical University, Shenyang, China; ${ }^{7}$ National Clinical Research Center for Laboratory Medicine, the First Affiliated Hospital of China Medical University, Shenyang, China; ${ }^{8}$ Collaborative Innovation Center for Diagnosis and Treatment of Infectious Diseases, Hangzhou, China; ${ }^{9} \mathrm{Key}$ Laboratory of AIDS Immunology, Chinese Academy of Medical Sciences, Shenyang, China; ${ }^{10}$ Kirby Institute, the University of New South Wales, Sydney, Australia

10.1136/sextrans-2021-sti.395 
Background The aim of this study was to develop and externally validate a prognostic model for survival in people living with HIV/AIDS (PLWHA) initiating ART based on two large population-based cohorts in China.

Approach The derivation cohort consisted of PLWHA treated between February 2004 and December 2019 in a tertiary center in Guangzhou, South China, and validation cohort of patients treated between February 2004 to December 2018 in another tertiary hospital in Shenyang, Northeast China. We assessed 20 candidate predictors for an endpoint of death from all causes. The prognostic model was developed from a multivariable cox regression model with predictors selected using the least absolute shrinkage and selection operator (Lasso). To assess the model's predictive ability, we quantified the discriminative power using the concordance (C) statistic and calibration accuracy by comparing predicted survival probabilities with observed survival probabilities estimated with the Kaplan-Meier method.

Outcomes The derivation cohort included 16481 patients and the external validation cohort comprised 5751 participants. The final model included 10 predictors: age, body mass index, route of HIV acquisition, coinfection with tuberculosis, coinfection with hepatitis C virus, haemoglobin, CD4 cell count, platelet count, aspartate transaminase, and plasma glucose. The C-statistic was 0.84 (95\% confidence interval 0.82-0.85) in internal validation after adjustment of optimism and 0.84 $(0.82-0.87)$ in external validation, which remained consistently above 0.75 in all landmark time points within five years of follow up when using time-updated laboratory measurements. The calibration accuracy was satisfactory in both derivation and validation cohorts.

Innovation and Significance We developed and externally validated a model to predict long-term survival in PLWHA on ART, which could be applied to individualized patient counseling and management during treatment, and future innovative trial design.

\section{P349 DEMOGRAPHIC AND BEHAVIOURAL CHARACTERISTICS OF PATIENTS DIAGNOSED WITH EARLY VERSUS LATE SYPHILIS AT WESTERN SYDNEY SEXUAL HEALTH CENTRE, 2015-2019}

${ }^{1} Y$ Hughes*, ${ }^{1} \mathrm{C}$ Chung, 1,2,3 Zablotska-Manos, 1,2,3 D Lewis. 'Western Sydney Sexual Health Centre, Parramatta, Australia; ${ }^{2}$ Westmead Clinical School, Westmead, Australia; ${ }^{3}$ Marie Bashir Institute for Infectious Diseases and Biosecurity, Westmead, Australia

10.1136/sextrans-2021-sti.396

Background Western Sydney Sexual Health Centre (WSSHC) provides a free sexual health service for its culturally-diverse population. As local syphilis incidence is increasing, we determined demographic and behavioural characteristics associated with early syphilis infection at WSSHC.

Methods We conducted a 5-year retrospective study of WSSHC's syphilis cases (2015-2019). Patient and laboratory data were extracted from medical records for analysis. The study definition of early syphilis (ES) included cases of primary syphilis, secondary syphilis, early latent infection $(<2$ years) and latent syphilis of unknown duration (VDRL titre $\geq 1: 4$ ). Late syphilis (LS) included both cases acquired $>2$ years previously and latent syphilis cases of unknown duration (VDRL titre $\leq 1: 2$ ). Statistical methods employed the Chisquare test for trend (diagnoses over time) and univariate/ multivariate logistic regression analyses to identify ES-associated variables. The study received local Ethics Committee approval.

Results 557 cases of syphilis, including 380 (68\%) ES and 177 (32\%) LS cases, were diagnosed in 553 individuals (454 men, 99 women). Syphilis diagnoses increased over time; early syphilis cases increased $26 \%$ annually $(\mathrm{p}<0.001)$. Median age (years) was $32(\mathrm{IQR}=19-17)$ for $\mathrm{ES}$ patients and 39 $(\mathrm{IQR}=20-86)$ for LS patients. ES patients, when compared with LS patients, were more likely to be male $(338 / 380$ [89\%] vs. $120 / 177$ [68\%];OR 3.82, 95\%CI=2.44-6.00), <35 years old (226/286 [79\%] vs. 154/271 [57\%];OR 2.86, 95\% $\mathrm{CI}=1.97-4.15)$, Australia-born (213/380 [56\%] vs. 43/177 [24\%];OR 3.97, 95\%CI $=2.67-5.92)$, previously infected with syphilis $(66 / 380 \quad[17 \%]$ vs. $10 / 177 \quad$ [6\%];OR $3.51, \quad 95 \%$ $\mathrm{CI}=1.76-7.01)$, chemsex-users $(72 / 380$ [19\%] vs. 4/177 [2\%]; OR 10.11, 95\%CI=3.63-28.17), PrEP-users (70/327 [21\%] vs. $6 / 170 \quad[4 \%] ; \mathrm{OR} \quad 7.44,95 \% \mathrm{CI}=3.16-17.54)$ and $\mathrm{HIV}$ coinfected $\quad(53 / 380 \quad[14 \%] \quad$ vs. $7 / 177 \quad$ [4\%];OR $4.83, \quad 95 \%$ $\mathrm{CI}=2.14-10.89$ ). Multivariable regression modelling indicated that these same ES-associated variables remained significant in men $(\mathrm{p}<0.05)$.

Conclusions Male gender, age $<35$ years, Australia-born, use of chemsex or PrEP, HIV co-infection and previous syphilis infection are risk factors associated with ES at WSSHC.

\section{P350 INFLUENCE OF THE COVID-19 PANDEMIC ON THE EPIDEMIOLOGY AND RESISTANCE OF NEISSERIA GONORRHOEAE IN AUSTRIA}

U Fuernkranz*, M Haller, A Stary. Pilzambulatorium Schloesselgasse, Vienna, Austria

\subsection{6/sextrans-2021-sti.397}

Background Gonorrhoea is a health threat, infection levels and drug resistances increased over the last decades. The COVID19 pandemic that hit Austria in 2020 led to several lockdown events such as closing of bars, clubs, hotels and the order to stay at home. The current study was designed to elucidate the influence of these measures in order to curtail the pandemic on the number of $\mathrm{N}$. gonorrhoeae diagnosed and their respective resistance patterns.

Methods Retrospective data of patients positive for N. gonorrhoeae and resistances to ceftriaxone, cefixime, azithromycin and penicillin were compared using data from 2019 and 2020 collected in and sent to the Outpatients Centre for Infectious Venero-dermatological Diseases in Vienna. Resistance testing was carried out by means of the Etest ${ }^{\circledR}$; MIC values were interpreted according to EUCAST guidelines.

Results Numbers of patients tested positive for N. gonorrhoeae declined dramatically from 639 in 2019 to 381 in 2020. The most prominent reduction was during the first lockdown (March to May 2020), with half as many gonococcal infections compared to this period in 2019. The second lockdown (November/December 2020) did not result in significantly lower numbers of gonococcal infected patients. In both years, no ceftriaxone-resistant isolate was detected. However, $2.3 \%$ of isolates from women showed borderline susceptibility (MIC $=0.094$ and $0.125 \mu \mathrm{g} / \mathrm{ml}$ ), compared to $0.4 \%$ of isolates from men. During the two years of evaluation, cefixime and azithromycin resistant strains decreased $(4 \%$ to $0 \%$ and $19 \%$ to $7 \%$, respectively), while penicillin-resistant strains increased from $9 \%$ to $20 \%$. 